\title{
Variation and variability in Drosophila grooming behavior
}

\author{
Joshua M. Mueller ${ }^{\mathrm{a}, \mathrm{b}, 1}$, Neil Zhang ${ }^{\mathrm{c}}$, Jean M. Carlson ${ }^{\mathrm{a}, \mathrm{b}}$, and Julie H. Simpson ${ }^{\mathrm{a}, \mathrm{c}, 1}$ \\ ${ }^{a}$ Interdepartmental Graduate Program in Dynamical Neuroscience, University of California, Santa Barbara; ${ }^{\mathrm{b}}$ Department of Physics, University of California, Santa Barbara; \\ ${ }^{\mathrm{c}}$ Department of Molecular, Cellular, \& Developmental Biology, University of California, Santa Barbara
}

\begin{abstract}
Behavioral differences can be observed between species or populations (variation) or between individuals in a genetically similar population (variability). Here, we investigate genetic differences as a possible source of variation and variability in Drosophila grooming. Drosophila grooming behavior confers survival and social benefits. Although the leg movements that constitute the grooming sequence are stereotyped, their order is not fixed. Grooming features of five drosophilid species exposed to a dust irritant were analyzed. Components of grooming behavior were conserved between and within species. However, significant differences in grooming syntax were identified, corresponding both to anterior and posterior grooming actions. Genetic heterogeneity was not found to be related to grooming variability, as melanogaster flies bred to increase genetic heterogeneity did not exhibit increased variability in grooming syntax. Likewise, no relationship between decreased heterogeneity and variability was identified. Finally, individual melanogaster flies were observed on consecutive days to determine the degree of variability of grooming behavior within an individual over time. Individual flies were not found to possess strong, stable grooming traits over several recordings. Additionally, standardization of sensory input using optogenetics did not eliminate grooming variability. In aggregate, these data suggest the importance of sensory inputs and other factors such as life history in grooming variability.
\end{abstract}

variation | variability | Drosophila | grooming syntax | computational ethology or over a century, researchers have understood that phenotypic variation arises from genotypic differences between organisms (1). Animal behavior is a phenotype partially under genetic control, so a sizable research effort has been dedicated to uncovering specific genes associated with observable differences in behavior between and within species (2). Different mouse species exhibit variation in monogamous behavior and parental care and different fly species show variation in courtship song $(3,4)$. Within a species, natural variability produces individual mice that differ in aggression and flies that implement different foraging strategies $(5,6)$. Mutant screens have also uncovered genes associated with differences in locomotion, courtship routines, and sleep patterns, among other complex behaviors (2, 7-9).

Fruit flies live in dirty environments, from laboratory vials to rotting fruit, and perform grooming actions to remove accumulated particulate. This behavior has been observed in several drosophilid species and is important for social behavior and survival (10-12). Past work has demonstrated that the leg movements used in grooming are stereotyped, but that sequences of actions are probabilistic and variable as opposed to fixed. However, the rules, or syntax, underlying grooming exhibit observable structure $(13,14)$. Different sensory experiences and life histories may influence probabilistic grooming behavior, leading us to ask: how much variation and variability in fruit fly grooming is due to genetics?

To address this question, $N=390$ flies were covered in dust and their grooming behavior was recorded for approximately 30 minutes each (Figure 1A). We analyzed flies from five drosophilid species (melanogaster, santomea, sechellia, simulans, and erecta), which are genetically distinct and inhabit different ecological niches. We also examined four common melanogaster stocks (Canton-S, Oregon-R, Berlin-K, and w1118), and several isogenic lines derived from parent stocks in the laboratory. To analyze this large data set, we used tools from computational ethology (15). An automated behavioral recognition system (ABRS, (16)) was used to classify fly behavior into one of five grooming actions (front leg cleaning, head grooming, abdomen grooming, back leg cleaning, wing grooming) and two non-grooming actions (walking and standing). After generating ethograms (behavioral time series records) for each fly, several grooming features were extracted (Figure 1B). Measures of central tendency and spread in the distributions of these features were calculated for each group and classification analysis was performed to determine the degree of separability between groups.

We found that several features of grooming are conserved across different drosophilid species. Automated classifiers labeled actions with high accuracy for all species, indicating that the movement primitives that make up grooming are stereotyped. Grooming sequences were probabilistic, with no clearly identifiable long repeats of actions. Additionally, flies tended to groom anterior body parts before posterior portions.

\section{Significance Statement}

Broadly speaking, genes influence behavior, but genes also play a role in determining the natural range of behavioral variability. Here, we show that Drosophila species exhibit differences in grooming behavior both between and within species. In particular, we demonstrate that transitions between grooming actions differ significantly between drosophilid species and common melanogaster stock lines, suggesting that these actions are under partial genetic control. Within melanogaster, however, genotype had no observable effect on the range of grooming behavior. This work establishes similarities in grooming behavior between drosophilids while also highlighting important differences, providing targets for future explorations of genetic, sensory, and developmental contributions to behavior.

Experimental design: J.H.S. Data collection: J.H.S., N.Z.; data analysis: J.M.M.; manuscript writing J.M.M.; manuscript editing: J.M.M., J.H.S., J.M.C.

The authors report no conflicts of interest.

${ }^{1}$ J.M.M. and J.H.S. contributed equally to this work.

${ }^{2}$ To whom correspondence should be addressed. E-mail: jmueller@ucsb.edu, jhsimpson@ucsb.edu
26 
We evaluated different grooming features by comparing their average values between groups and their range within groups, using the concepts of variation and variability as defined by Ayroles et al. (9). Variation refers to differences in phenotype between genetically distinct populations, such as drosophilid species. For example, inter-species variation in drosophilid larval digging behavior has been identified based on the duration distributions of "dives" into an agarose substrate (17).

In contrast, variability describes how a trait differs within a genetically similar population. Intra-species variability has been characterized in the locomotor behavior of fruit flies, as some populations exhibit a wide range of locomotor behaviors, whereas others exhibit a narrower range (9).

Here, inter-species comparisons were used to look for variation in grooming features (such as syntax and action duration distributions) between flies with large genetic differences. Several significant differences in syntax, largely related to posterior grooming actions, were identified, allowing for highly accurate species-specific classification.

Next, we looked for variation within melanogaster stock lines using similar analysis. Although grooming features were more similar between melanogaster stocks than between species, stock lines did exhibit detectable differences in grooming, largely related to overall grooming activity levels (e.g. after dusting, some lines spend a large proportion of time grooming, while others walk or stand more). Notably, male and female flies within a stock line also differed in grooming syntax and activity levels.

We then hypothesized that if a heterogeneous gene pool contributes to variability in grooming behavior, it should be possible to observe trends in variability between melanogaster populations with high and low genetic diversity. Starting from different commonly used stocks, flies were interbred for several generations to produce flies with high genetic variability and, in parallel, individuals were isogenized to minimize genetic variability. All three groups exhibited similar variability in measured grooming features.

Furthermore, flies tested on sequential days revealed that between-fly differences in syntax were similar to within-fly differences over several sessions. Finally, flies stimulated using optogenetic manipulation to induce grooming exhibited increased stereotypy in grooming. However, within-individual grooming variability between stimulation sessions was not fully abolished. Together, these data suggest that, in addition to genetics, several factors, including sensory experience, developmental stochasticity, and noisy circuit dynamics may contribute to grooming variability

\section{Results}

Drosophilids exhibit a robust grooming response but different syntax after irritant exposure. Regardless of genotype, all flies possessed several common grooming features. Here, five grooming actions were observed consistently across all five species of drosophilids (erecta, sechellia, santomea, simulans, melanogaster), indicating a conserved behavioral response. In this analysis, a single stock line (Canton-S, N=18) was used as the representative melanogaster group.

To quantify the behavioral response to dusting, the proportion of time spent grooming (as opposed to walking and standing) was calculated for each fly (Figure 2A). The groom- ing distributions between species were all statistically different (Wilcoxon rank-sum test, $p<.05$ after Bonferroni correction), but all species had median grooming time proportions greater than $35 \%$.

Grooming proportions were then examined in more detail by considering all seven actions and the progression of those actions over time, as in Figure 1B. These species exhibited a qualitatively similar grooming progression characterized by initial anterior grooming followed by increased posterior grooming, walking, and standing, but the relative proportions and timing of behaviors over time differed (Supplementary Information).

Grooming syntax (the transition probabilities between discrete behaviors) was calculated from the ethogram of each dusted fly $(N=390)$. With seven behavioral states, 42 transitions were possible, excluding self-transitions. Thus, syntax was represented as a 42-dimensional vector for subsequent classification analysis and visualization. Grooming syntax across all flies was probabilistic with high transition probabilities within anterior grooming actions (front leg cleaning, head grooming) and posterior grooming actions (abdomen grooming, back leg cleaning, wing grooming). The average syntax for each species is illustrated as a weighted, directed graph in Figure 2.

Finally, action duration distributions were calculated from ethograms. Distributions of grooming actions were similar across species and had probability peaks between 500 and 750 ms (Supplementary Information).

Several significant differences in grooming syntax between melanogaster and non-melanogaster species were identified. To compare syntax, transition probability distributions for each action transition were compared between species in a pairwise manner. 38 of 42 unique syntax elements (90.5\%) were significantly different between at least two species (Wilcoxon rank-sum test, $\mathrm{p}<0.05$ after Bonferroni correction). Overall, 119 of $420(28 \%)$ of pairwise syntax comparisons revealed differences between species.

Of these syntactic differences, $71(60 \%)$ occurred between melanogaster and non-melanogaster species. In particular, posterior motif grooming transitions (i.e. transitions between abdomen grooming, back leg rubbing, and wing grooming) were consistently significantly different, on average, as were transitions between back leg rubbing, standing, and walking. Figure 2B illustrates these syntactic differences.

Figure $2 \mathrm{C}$ depicts a low-dimensional embedding of species syntax using t-SNE. This visualization suggests that different species possess distinguishable syntax, as points are aggregated by species. Classification and clustering analysis was applied to species syntax to verify this interpretation.

Logistic regression classified flies by species according to syntax with $>90 \%$ accuracy in 9 of 10 pairwise comparisons (Figure 2D). K-medoids clustering reinforced this finding, as these groups were classified with $>90 \%$ accuracy using fewer than 20 syntax elements (Supplementary Information). Classification according to progressions and grooming proportions achieved similar performance, but using bout durations performed the worst (Supplementary Information).

melanogaster strains exhibit variation in grooming behavior. Standard lab strains (Canton-S, Berlin-K, Oregon-R, w1118) were analyzed for differences in grooming proportions, progression, syntax, and bout duration distributions. Groom- 
A

BETWEEN

$\mathrm{SPECIES} \rightarrow$ erecta santomea sechellia simulans $(\mathrm{N}=16) \quad(\mathrm{N}=18) \quad(\mathrm{N}=15) \quad(\mathrm{N}=16)$
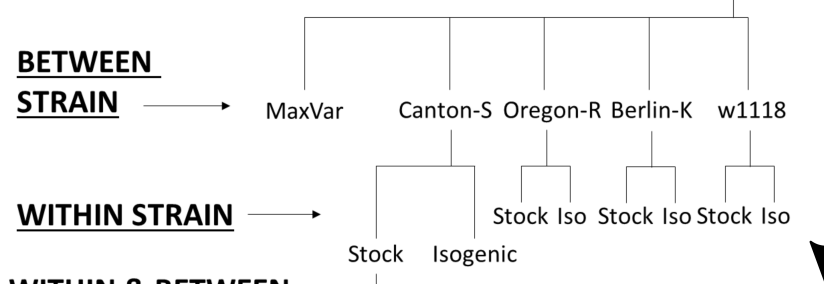

WITHIN \& BETWEEN INDIVIDUAL $\longrightarrow 15$ individuals over 3 days

B $(\mathrm{N}=45)$
Genetic heterogeneity (high to low)

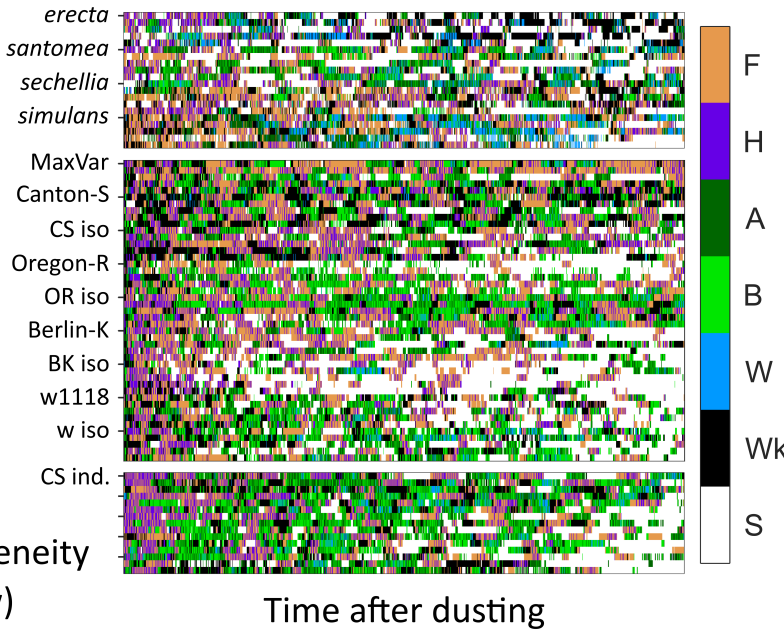

Grooming syntax

Bout duration distributions

Grooming progression

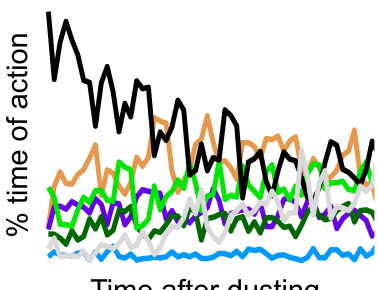

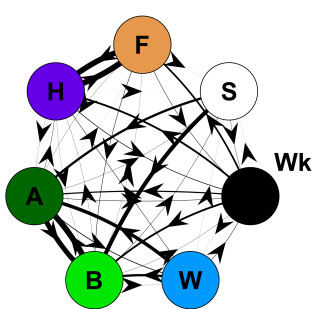

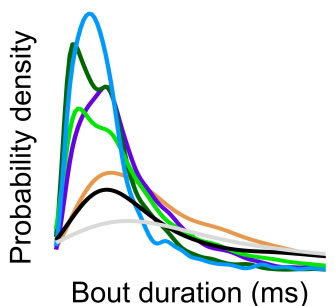

Fig. 1. Grooming variability dataset and analysis overview. A. In total, $N=390$ flies were dusted and their grooming activity was recorded for approximately half an hour each. Five drosophilid species, four melanogaster stock lines, one interbred melanogaster line, and six isogenic melanogaster lines were analyzed for similarity, differences, and variability in grooming behavior. On the left is a schematic of the different drosophilid groups included in this analysis. Higher levels of the tree indicate higher levels of genetic diversity (scale is relative, not absolute). On the right is a sample of ethograms generated by automated annotation of grooming video. Color indicates the occurrence of the five grooming actions $(F=$ front leg cleaning, $\mathrm{H}=$ head grooming, $\mathrm{A}=$ abdomen grooming, $\mathrm{B}=$ back leg cleaning, $\mathrm{W}=$ wing grooming), Wk $=$ walking, and $\mathrm{S}=$ standing. $\mathrm{B}$. Features scored from ethograms provide summary representations of grooming behavior. The proportion of time spent grooming, overall or over time, provides the coarsest description of the behavioral response to a dust stimulus. Regardless of genotype, all flies exhibit probabilistic (not fixed) action sequences consisting of the same set of five grooming actions, walking, and standing after exposure to irritant. Additionally, most flies follow a typical grooming progression pattern: initial anterior grooming followed by increased posterior grooming. The amount and timing of walking and standing, however, can vary significantly between flies. Grooming transitions (syntax) and action (bout) duration distributions provide representations of individual grooming decisions within a recording session. Shown here are example features scored from Canton-S flies.

ing syntax differed between stocks, allowing for classification above chance levels. Grooming progressions were also different between stocks. Overall grooming proportions and bout durations achieved the worst classification performance, indicating that these features are more highly conserved.

The grooming syntax of melanogaster stocks is depicted in Figure 3A. Similar to the species analysis, all action transition probability distributions were compared in a pairwise manner to look for variation in syntax. Only 19 of 42 unique syntax element comparisons (45\%) differed significantly between any two stocks and, of these, only two within-motif transition (both posterior motif) differed significantly. Within-motif syntax elements are of particular interest because they represent the most common, most highly stereotyped action transitions observed across flies of all genotypes (see Figure 2A and B for visualizations of these transitions). The other significantly different transitions mostly involved transitions to and from walking and standing (Supplementary Information). However, these differences allowed for classification of stocks above chance, as illustrated in Figure 3A.

Examination of other grooming features revealed that over- all grooming proportions could also account for differences observed between stocks. Figure 3B shows a ternary plot of action proportions, showing that Canton-S flies spend more time walking than other stocks. Relatedly, the syntax element exhibiting the greatest statistically significant difference was the wing grooming to walking transition, shown in the middle of Figure 3A. Classification accuracy was poor when using grooming proportions but comparing grooming progressions, which are similar to proportions but account for changes in grooming behavior over time, yielded significant differences.

Canton-S flies' higher propensity to walk after grooming their wings is reflected both in their syntax and grooming proportions, resulting in similar classification accuracy (Figure $3 \mathrm{~B}$, right) whether using syntax or progressions. Within Canton-S, activity levels also separated male and female flies, as male flies tended to walk more than females (Supplementary Information).

Figure 3B also illustrates the high degree of variability in melanogaster syntax. The wing grooming to back leg cleaning transition exhibited the largest difference between median values of any syntax element (comparison of Canton-S and 
A

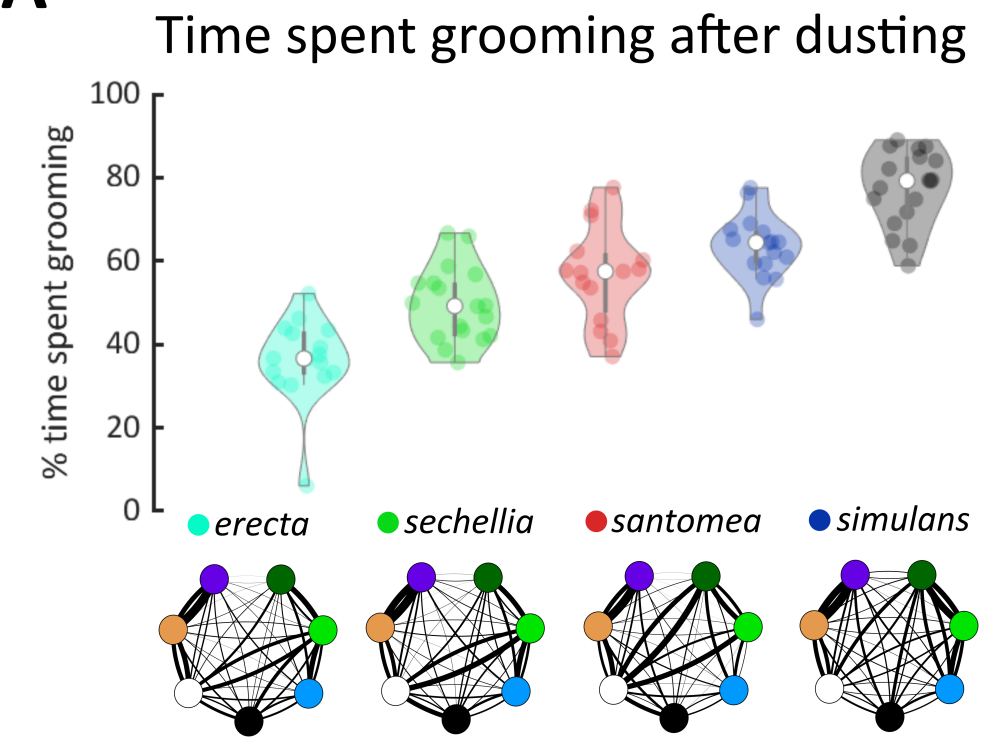

Low-dimensional embedding (t-SNE) of grooming syntax

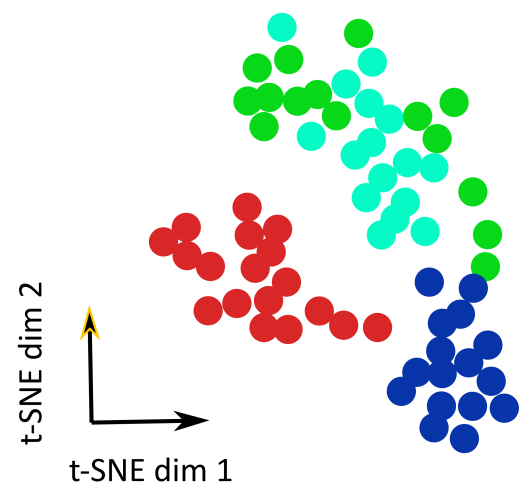

B

Group average syntax

\section{- melanogaster}

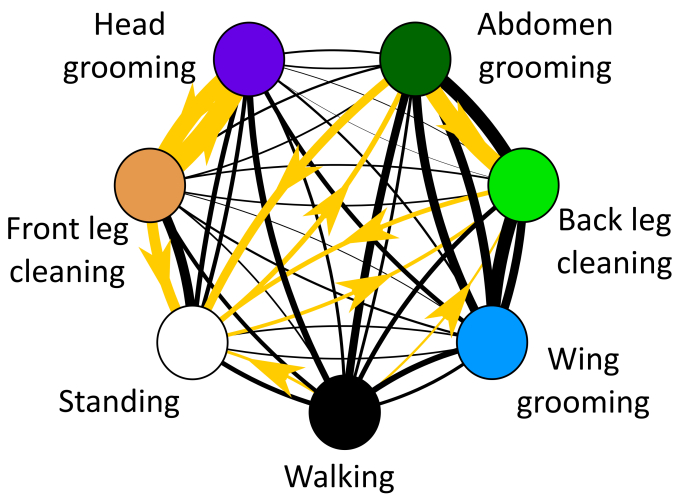

D

\section{Syntax classifier performance}

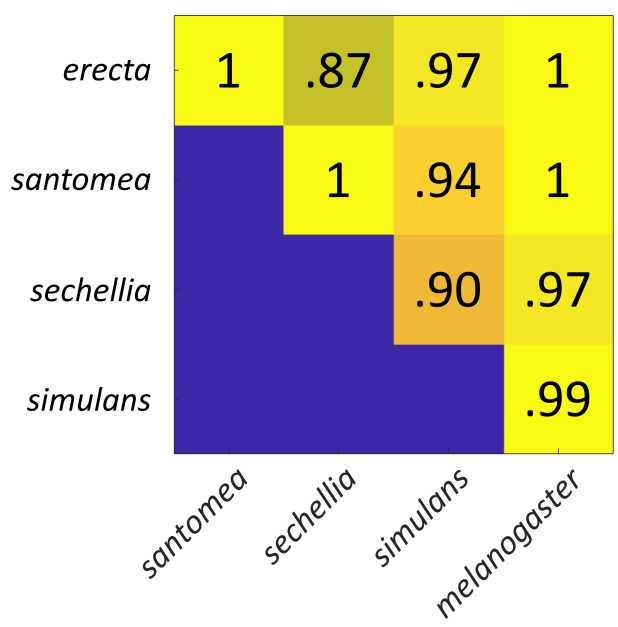

Fig. 2. Drosophila species share grooming features but exhibit between-species variation in grooming syntax. A. Dusting elicits a conserved grooming response across drosophilids. These species produce a probabilistic grooming sequence (as shown in Figure 1A), which can be characterized by the transition probabilities (syntax) between grooming actions (as represented in Figure 1B, calculated as in as in (14)). Shown are violin plots of grooming proportions for each species examined here. Colored points represent a single fly, with color indicating species (as indicated by points next to species names in A and B). White points indicate the median value and plot with indicates density. B. Several syntax elements differ between drosophilid species. The mean syntax for each species is depicted as a graph, with nodes representing grooming actions and edges indicating transition probability. Thicker edges indicate higher probabilities. On the melanogaster syntax graph, the 10 action transitions exhibiting the largest magnitude differences between melanogaster and non-melanogaster species are highlighted in gold. These differences are identifiable in anterior motif transitions, which use the front legs to perform grooming actions. Species also differ in their transitions between posterior grooming actions and non-grooming actions (walking and standing) $\mathrm{C}$. Each fly's 42-dimensional syntax vector was plotted in two dimensions after dimensionality reduction using t-SNE. t-SNE preserves local distance structure, indicating that tightly grouped clusters of points are similar to one another. In this case, dimensionality reduction reveals that drosophilid species exhibit significant differences in grooming syntax, as syntax vectors congregate by color. D. Classification analysis confirms the qualitative clustering observed in C. Shown is a heat map of accuracy rates of pairwise logistic regression classifiers trained on syntax vectors. Samples used for classification were chosen to be the same size, so classification at chance would be 0.5 . Consistent classification accuracy values $>.9$ indicate that species are highly separable by syntax.

Oregon-R yielded this difference), but none of these distributions possessed detectable statistical differences due to their concomitantly large variances.

\footnotetext{
Grooming behavioral variability is similar across melanogaster genotypes. To examine the relationship
}

between genetic heterogeneity and behavioral variability, each melanogaster lab strain was compared to lines bred to maximize genetic heterogeneity (MaxVar) and minimize genetic heterogeneity ("isogenic" lines). To quantify this, the variances of grooming transition probability distributions were calculated and compared. 
bioRxiv preprint doi: https://doi.org/10.1101/2020.08.15.252627; this version posted August 15, 2020. The copyright holder for this preprint (which was not certified by peer review) is the author/funder, who has granted bioRxiv a license to display the preprint in perpetuity. It is made available under aCC-BY-NC 4.0 International license.

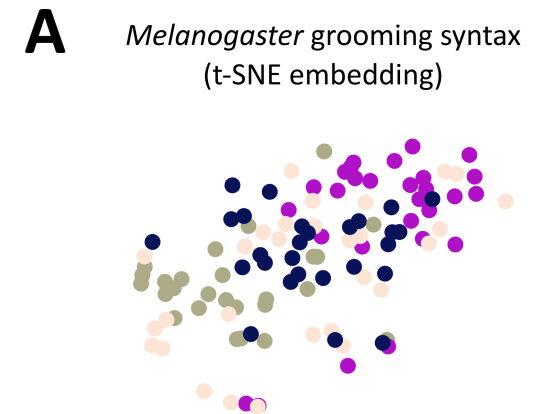

Canton-S

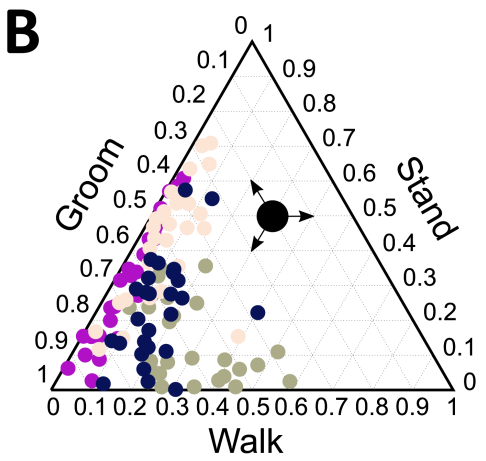

Wing grooming to walking

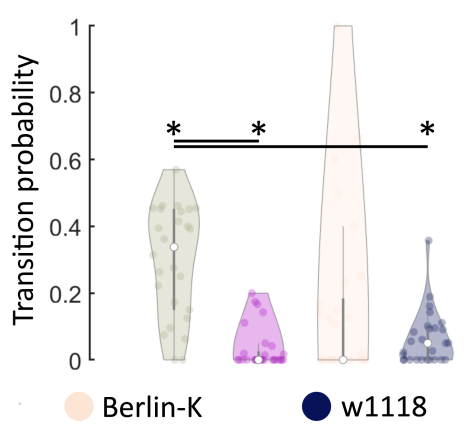

Wing grooming to back leg cleaning

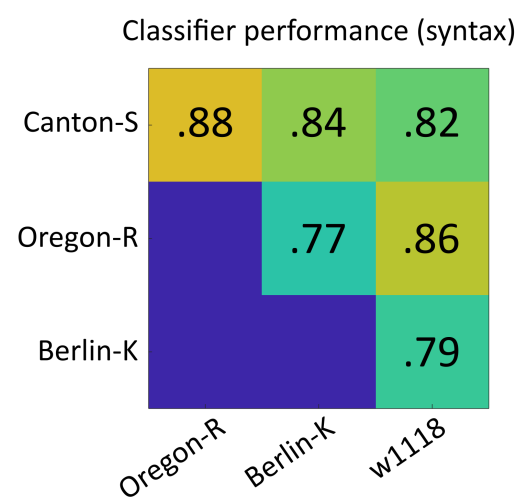

Classifier performance (progressions)
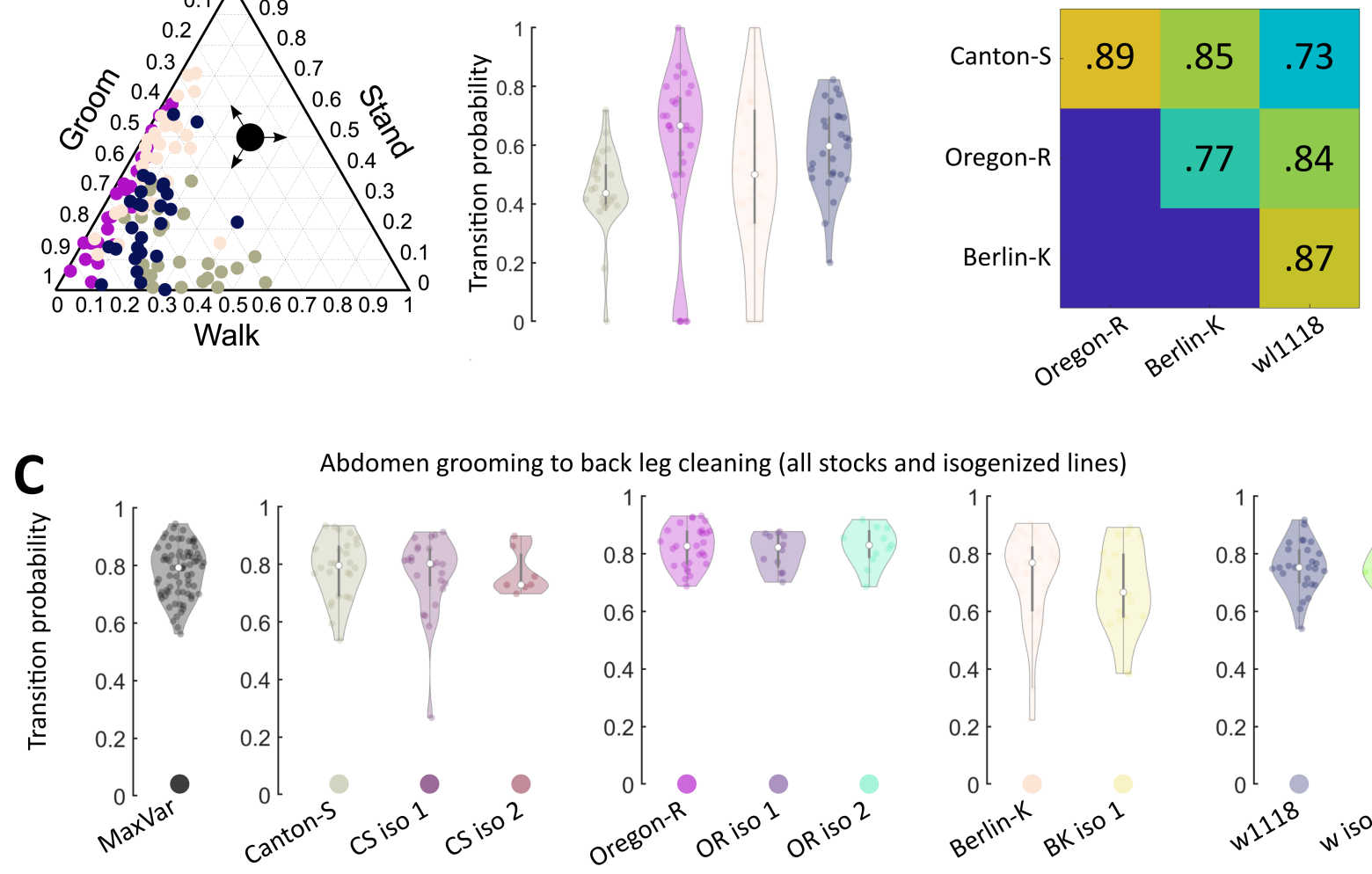

Fig. 3. Within melanogaster, different stocks exhibit syntactic differences and different activity levels. Genetic homogeneity does not correspond to behavioral stereotypy. A. melanogaster stocks exhibited variation in grooming syntax, though many features were shared. This is illustrated by the high degree of overlap in the t-SNE plot of grooming syntax vectors (left). The middle panel displays distributions of one representative strong grooming transition; $215 / 252$ (85.3\%) of transition distributions were not significantly different from one another. Of the 19 transitions that were significantly different, only two were within-motif transitions; the other significantly different transitions mostly involved transtions to and from walking and standing (Supplementary Information). Due to several syntactic differences between stocks, however, classifier analysis was able to distinguish between stocks with greater than $75 \%$ accuracy (right). B. Most syntax elements are similar between melanogaster stocks, but Canton-S flies walked more than other stocks. On the left is a ternary plot of grooming, walking, and standing proportions for each species. Colored points represent individual flies. The large black point with arrows indicates how to read activity proportions; the example point corresponds to $20 \%$ grooming, $30 \%$ walking, and $50 \%$ standing. Due to differences in activity levels, some walking-related syntax elements differed between Canton-S flies and other stocks. The middle plot illustrates the most significantly different syntax element between stocks (Wilcoxon rank-sum test, $p<.05$ corrected for multiple comparisons). Classifier performance on action proportions (right) is similar to performance on syntax, indicating that syntactic differences are not a significantly more informative source of variation between stocks than activity levels. C. Genetic homogeneity did not correspond to behavioral variability. Shown are the distributions of wing to back leg transitions for a line bred for maximum genetic heterogeneity (MaxVar), each stock line, and lines derived from stocks to minimize genetic heterogeneity (iso). MaxVar flies did not exhibit a higher degree of variability than stock lines. Isogenized lines did not exhibit a lower degree of variability than their parent stocks.

Only $6 / 252(2.4 \%)$ transition probability distributions possessed statistically significantly different variances between MaxVar, Canton-S, and the isogenic lines out of all possible pairwise comparisons (Levene's test, $p<.05$ after Bonferroni correction). Moreover, none of these differences corresponded to within-motif transitions, indicating that variability of common transitions is similar regardless of genetic heterogeneity in a population. These findings were similar for Oregon-R 
(8/252), Berlin-K (18/108), and w1118 (2/108) stock and isogenic comparisons.

Figure $3 \mathrm{C}$ provides the transition probability distributions for the most common posterior motif transition (abdomen grooming to back leg cleaning) for all stocks and stock-derived isogenic lines. This transition exhibits wide variability in many populations and even populations with smaller variability (CS iso 2) are not different enough to achieve statistical significance after accounting for multiple hypothesis testing.

Finally, we analyzed 15 Canton-S flies that were recorded over three consecutive days after irritant exposure. In this experiment we aimed to isolate the magnitude of grooming variability that is due to differences in sensory experience (since the dusting protocol does not allow for perfect replication of sensory experience) and life history (since flies will have been exposed to the same irritant several times by the end of the experiment). Ethograms from three example flies are provided in Figure 4A.

Flies did not exhibit strong longitudinal grooming trends, as neither the total amount of grooming nor the time to completion of $50 \%$ of their total grooming decreased between the first and third days of the experiment (Supplementary Information). Additionally, intra-individual variability in syntax across three sessions was of the same magnitude as inter-individual variation in syntax, suggesting that non-genetic factors account for a significant proportion of grooming variability (Supplementary Information).

Standardizing sensory experience does not abolish grooming behavioral variability. To further probe the sensory basis of within-individual variability, 10 Bristle-spGAL4-1 > CsChrimson flies (Bloomington Stock Center, RRID: BDSC_71032) were stimulated using optogenetic manipulation to induce anterior grooming (as in (18)). Figure 4B provides ethograms from this experiment, with red bars indicating the two stimulation windows. Even when sensory experience was controlled using optogenetic stimulation, flies exhibited variability in their grooming response. Grooming stereotypy was quantified using the entropy rate of the grooming syntax, represented as a first-order Markov chain (see Supplementary Methods for details). Briefly, a Markov entropy of 0 indicates complete predictability and stereotypy, while higher values indicate more random action transition structure.

The entropy rate for optogenetically stimulated flies was lower than for dusted flies, indicating a higher degree of stereotypy in grooming (Figure 4C). We nonetheless observed withinindividual variability between stimulation windows, indicating that standardization of sensory input does not fully abolish grooming variability. Figure $4 \mathrm{C}$ illustrates this finding, as the differences in entropy between sessions for three example flies are highlighted using red lines.

\section{Discussion}

Here, we analyzed the grooming behavior of 390 flies, including five different drosophilid species and four common melanogaster stocks, to investigate the relationship between genetic heterogeneity, variation, and variability in grooming behavior. Large genetic differences (species-level) correspond to identifiable differences in several grooming features. Within melanogaster, stock lines also exhibited variation in grooming syntax and progressions. However, increased genetic hetero- geneity did not correspond to increased behavioral variability. Analysis of 15 Canton-S flies recorded over consecutive days found no evidence for individuality in grooming behavior, as all flies possessed highly similar grooming features across all days. Optogenetically stimulated flies also exhibited intraindividual variability in grooming behavior. Taken together, these results demonstrate that large genetic differences result in distinguishable grooming phenotypes (variation), but that genetic heterogeneity within a population does not necessarily correspond to an increase in the range of grooming behavior (variability). These findings suggest that differences in sensory experience and developmental history likely account for grooming variability within a population.

Genetic influences of behavioral variation and variability. In a classic example of behavioral variation, fruit fly larvae expressing the rover and sitter alleles of the foraging gene exhibit, respectively, greater or lesser propensities to leave patches of food $(6,7,19)$. Several genes that affect fruit fly grooming behavior have been identified in mutant screens (20), but the degree of normal variability due to genetic factors has not been characterized.

Here, we identified significant inter-species variation in grooming syntax, suggesting a genetic basis for group differences in grooming behavior. Some species differ from melanogaster in their propensity to perform anterior grooming actions; the anterior motif actions are significantly less strongly coupled in non-melanogaster drosophilids, suggesting that anterior neuronal circuitry and sensory physiology may differ.

We also identified differences in grooming behavior between commonly used melanogster stock lines (Supplementary Figure 9). Male and female Canton-S flies also exhibited variation in grooming behavior attributable largely to differences in walking behavior, providing a target for characterizing potential developmental origins of differential grooming behavior.

We also examined whether greater genetic heterogeneity within a population corresponded to greater behavioral variability. The grooming syntax variability in a population of melanogaster flies bred to maximize genetic diversity was statistically equivalent to the variability of parent stocks and isogenic lines, suggesting that non-genetic factors contribute significantly to grooming variability.

Variability also encompasses individuality in animal behavior, typically defined as a trait-like feature that persists stably over several observations. Individuality has been identified in fruit fly turning decisions (21), mouse roaming behavior (22), and bumblebee foraging behavior (23), among others $(24,25)$, indicating the importance of considering within-individual variability, or the degree to which an individual's behavior varies in repeated trials. Our analysis found no evidence for individuality in grooming, as Canton-S flies exhibited similar degrees of within-individual variability and between-individual variation. One explanation for this observation is that, within a species, genetic influences on grooming behavior are outweighed by differences in sensory experience, which is controlled here to the greatest extent possible using standardized experimental protocols but some differences in sensory experience between trials is assumed to exist.

Environmental and stochastic influences of behavioral variation and variability. Our analysis of genetic contributions to 

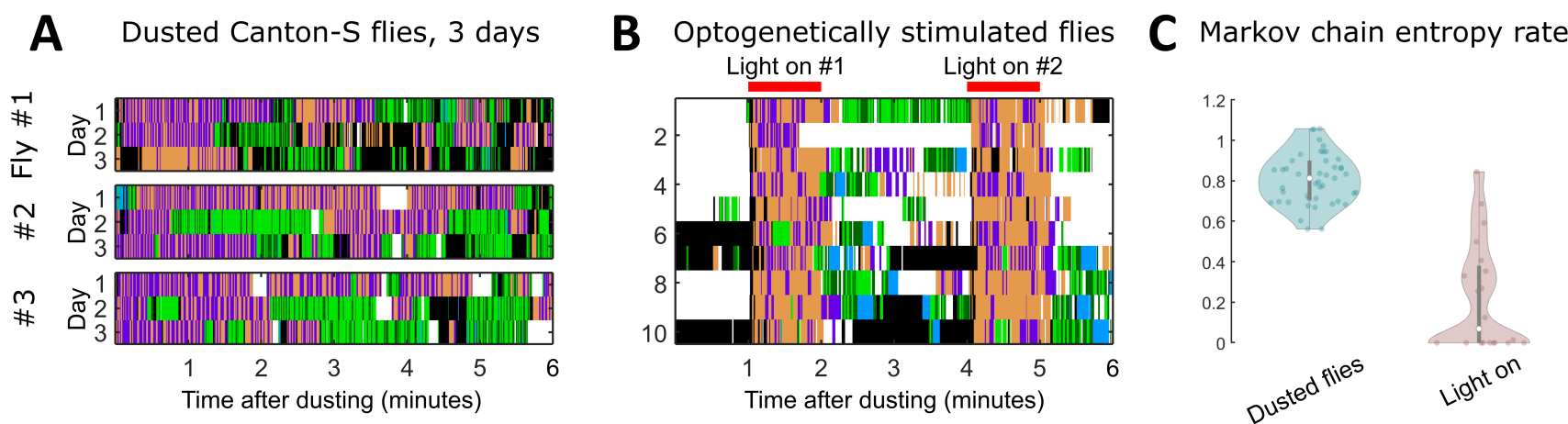

Fig. 4. Within-individual grooming differences suggest that non-genetic factors account for a significant portion of variability in behavior. A. Shown are portions of
ethograms from three Canton-S flies observed on consecutive days after dust irritant exposure. The differences in ethograms on consecutive days indicate that non-genetic
factors must account for some amount of within-individual grooming variability. B. Shown are ethograms of 10 Bristle-spGAL4-1 $>$ CsChrimson flies (18). Flies were
optogenetically stimulated to induce anterior grooming in two separate one-minute windows, indicated by the red bars. Between these windows, flies still exhibit within-individual
grooming variability even though the sensory experience is more uniform than repeated dust exposure. C. Markov chain entropy, a measure of grooming stereotypy, was
calculated from syntax. Optogenetically stimulated flies (right) exhibited lower entropies, corresponding to a higher degree of stereotypy, than dusted flies (left). However,
optogenetically stimulated flies exhibited differences in stereotypy between stimulation windows, implicating sources of grooming variability beyond genetic and sensory
influences.

behavioral variation and variability in the grooming suggests that at the species level, flies show significant differences in the grooming sequence, especially in the syntax of transition probabilities, that allow accurate classification. The extent of genetic differences among common lab wild-type stocks also support classification, but the accuracy is lower and the effect size of the differences is smaller. The grooming sequence is probabilistic in all species and stocks examined, indicating that this flexibility in action order may be a useful feature of the behavior. The extent of variability in sequence syntax does not correlate with genetic homogeneity, suggesting that other factors contribute. Difference in the initial sensory stimuli is a strong candidate source of variability, supported by our observations that the same fly shows sequence differences on subsequent trials and more uniform sensory stimulation with optogenetic control reduces sequence variability. Other potential sources of variability include developmental stochasticity (25), life history/experience/enrichment (26) and intrinsic noise in the neural circuits that control grooming. Future work will explore these alternative possibilities.

\section{Materials and methods}

Genetic stocks. Canton-S, Oregon-R, Berlin-K, w1118, BristlespGAL4-1 (R38B08-AD; R81E10-DBD), and 20XUASCsChrimson-mVenus (attp18) stocks were obtained from the Bloomington Stock Center. Isogenic (more accurately, reduced genetic variability) stocks were made by crossing single males to double-balanced stocks and then back-crossing males to the double balancer stock to isolate single second and third chromosomes. Single pairs were mated to reduce variability of $\mathrm{X}$ and IV. $\sim 2$ independent isogenic lines from each melanogaster stock were generated; note that many attempts to isogenize result in lethality, as anecdotally reported by colleagues. Maximum Variability stocks were obtained by crossing each melanogaster strain to double balancers and then crossing the progeny together and selecting against the balancers. This allowed combination of chromosomes for all four strains. The progeny were allowed to interbreed for several generations to enable recombination in the females. Full cross schemes available on request. Drosophilid species stocks were obtained from Tom Turner, UCSB, and the National Drosophila Species Stock Center (https://www.drosophilaspecies.com/).

Data collection and processing. Grooming was induced and analyzed as described in Zhang et al. 2020 and Seeds et al. 2014 (13, 18). Three chambers were used in fly dusting assay: dusting chamber (24 well Corning tissue culture plate \#3524), transfer chamber and recording chamber. Recording chambers were coated with Insect-a-slip (BioQuip Products Cat\#2871A) to discourage wall-climbing and cleaned daily. Dust-induced grooming assays were performed in $21-23^{\circ} \mathrm{C}$. 4-7 day old male flies were anesthetized on ice and transferred to the middle four wells of the transfer chamber. Flies were left in the transfer chamber for 15 minutes to recover. Approximately 5 mg Reactive Yellow 86 dust (Organic Dyestuffs Corporation CAS 61951-86-8) was added into each of the 4 middle wells of dusting chamber. For fly dusting, the transfer chamber was aligned with the dusting chamber. Flies were tapped into the dusting chamber and shaken 10 times. After dusting, flies and dust were transferred back into the transfer chamber. Transfer chamber was tapped against an empty pipette tip box to remove extra dust. Dusted flies were then immediately tapped into recording chamber for video recording. The entire dusting process was performed in a WS-6 downflow hood. Approximately 10 individuals were recorded for each genotype. $30 \mathrm{~Hz}$ videos were recorded for 50,000 frames (27.78 min) with a DALSA Falcon2 color 4M camera. A white LED ring right was used for illumination.

Optogenetic stimulation protocol is replicated from (18). Further details can be found in the Supplementary Methods. For each set of experimental comparisons (between species, within species, within individual), a single experimenter performed all dusting assays to eliminate experimenter-related differences that may arise.

Videos were processed through the Automated Behavior Recognition System (ABRS, (16)), trained on a classifier using melanogaster flies to generate ethograms. Grooming actions were described previously (27). ABRS was used to generate ethograms. Briefly, the raw video frames were pre-processed 
to generate 3-channel spatial-temporal images (ST images). Features were extracted in three timescales and saved into different channels of ST images: 1. raw frame; 2. difference between two frames; 3 . spectral features extracted from a 0.5 sec window. A convolutional network trained by ST images under different light conditions was then used to label the behavior identified in each frame. A different network was trained for classification of each species due to differences in body size and light conditions. All networks achieved $>70 \%$ validation accuracy, which is similar to the agreement between human-annotated ethograms.

Data analysis. All ethogram features were extracted using custom-written code in MATLAB 2019a. Grooming progression vectors were generated for each fly by calculating the proportion of each action in 10 non-overlapping windows (2.78 minutes each), yielding a 70-dimensional vector for each fly (10 windows with 7 behavioral proportions). Grooming syntax was defined as the first-order transition probabilities between actions. Syntax for each fly was calculated as described in (14). Bout duration distributions were generated as described in (14), using a normalized histogram with 20 bins of equal width for each behavior. Bin width was determined independently for grooming and non-grooming actions, as standing and walking exhibit longer tailed distributions than grooming actions. Thus, duration distribution vectors were 140-dimensional for each fly. Examples of progression, syntax, and duration distribution vectors can be found in the Supplementary Information.

Statistics for comparisons between grooming features were calculated using built-in MATLAB functions. K-medoids clustering, t-SNE, and logistic regression classification analysis were performed using built-in MATLAB functions (Supplementary Information).

\section{Acknowledgments}

Stocks obtained from the Bloomington Drosophila Stock Center (NIH P40OD018537) were used in this study. Thanks to Karen Hibbard, Barry Ganetzky, and Claire McKellar for consultations on isogenization strategies. Thanks to Jordan Zawaydeh for data collection of individual fly data. Thanks to Kristin Branson and Matthieu Louis for advice and comments on the manuscript. This work was also supported by HHMI, NIH R01NS110866 (JHS), the David and Lucile Packard Foundation and the Institute for Collaborative Biotechnologies through Contract No. W911NF-09-D-0001 from the U.S. Army Research Office (JMM and JMC).

\section{References.}

1. W Johanssen, The genotype conception of heredity. Int. J. Epidemiol. 43, 989-1000 (2014).

2. B Baker, B Taylor, J Hall, Are complex behaviors specified by dedicated regulatory genes? reasoning from drosophila. Genetics 105, 13-24 (2001).

3. A Bendesky, et al., The genetic basis of parental care evolution in monogamous mice. Nature 544, 434-439 (2017).

4. Y Ding, et al., Neural evolution of context-dependent fly song. Curr. Biol. 29, 1089-1099 (2019).

5. D Anderson, Circuit modules linking internal states and social behaviour in flies and mice. Nat. Rev. Neurosci. 17, 692-704 (2016).

6. A Allen, I Anreiter, M Neville, M Sokolowski, Feeding-related traits are affected by dosage of the foraging gene in drosophila melanogaster. Genetics 205, 761-773 (2017).

7. M Sokolowski, Drosophila: Genetics meets behavior. Nat. Rev. Genet. 2, 879-890 (2001).

8. B Gaertner, et al., Heritable variation in courtship patterns in drosophila melanogaster. G3 5 , 531-539 (2015).

9. J Ayroles, et al., Behavioral idiosyncracy reveals genetic control of phenotypic variability. PNAS 112, 6706-6711 (2015).

10. A Szebenyi, Cleaning behaviour in drosophila melanogaster. Anim. Behav. 17, 641-651 (1969).
11. B Spruijt, J van Hooff, W Gispen, Ethology and neurobiology of grooming behavior. Physiol. Rev. 72, 825-852 (1992).

12. M Zhukovskaya, A Yanagawa, B Forschler, Grooming behavior as a mechanism of insect disease defense. Insects 4, 609-630 (2013).

13. A Seeds, et al., A suppression hierarchy among competing motor programs drives sequential grooming in drosophila. elife 3, e02951. doi: 10.7554/eLife.02951 (2014).

14. J Mueller, P Ravbar, J Simpson, J Carlson, Drosophila melanogaster grooming possesses syntax with distinct rules at different temporal scales. PLoS Comp. Biol. 15, e1007105 (2019).

15. S Datta, D Anderson, $\mathrm{K}$ Branson, $\mathrm{P}$ Perona, $\mathrm{A}$ Leifer, Computational neuroethology: A call to action. Neuron 104, 11-24 (2019).

16. P Ravbar, K Branson, J Simpson, An automatic behavior recognition system classifies animal behaviors using movements and their temporal context. J. Neuro. Methods 326, 108352 (2019).

17. D Kim, M Alvarez, L Lechuga, M Louis, Species-specific modulation of food-search behavior by respiration and chemosensation in drosophila larvae. eLife 6, e27057 (2017).

18. N Zhang, L Guo, J Simpson, Spatial comparisons of mechanosensory information govern the grooming sequence in drosophila. Curr. Biol. 30, 988-1001 (2020).

19. J de Belle, A Hilliker, M Sokolowski, Genetic localization of foraging (for): A major gene for larval behavior in drosophila melanogaster. Genetics 123, 157-163 (1989).

20. R Phillis, et al., Isolations of mutations affecting neural circuitry required for grooming behavior in drosophila melanogaster. Genetics 133, 581-592 (1993).

21. S Buchanan, J Kain, B de Bivort, Neuronal control of locomotor handedness in drosophila. PNAS 112, 6700-6705 (2015).

22. J Freund, et al., Emergence of individuality in genetically identical mice. Science 340, 756759 (2013).

23. S Klein, C Pasquaretta, A Barron, J Devaud, M Lihoreau, Inter-individual variability in the foraging behaviour of traplining bumblebees. Sci. Reports 7, DOI:10.1038/s41598-017-049198 (2017).

24. S Takagi, R Benton, Animal behavior: A neural basis of individuality. Curr. Biol. 30, R698R720 (2020).

25. G Linneweber, et al., A neurodevelopmental origin of behavioral individuality in the Drosophila visual system. Science 367, 1112-1119 (2020).

26. J Akhund-Zade, S Ho, C O'Leary, B de Bivort, The effect of environmental enrichment on behavioral variability depends on genotype, behavior, and type of enrichment. J. Exp. Biol. 222, jeb202234. doi:10.1242/jeb.202234-R12 (2019).

27. S Hampel, R Franconville, J Simpson, A Seeds, A neural command circuit for grooming movement control. eLife 7, e08758 (2015). 


\section{BETWEEN}

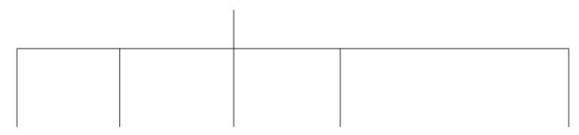

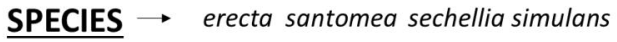

$$
(N=16) \quad(N=18) \quad(N=15) \quad(N=16) \quad(N=325)
$$

BETWEEN

STRAIN
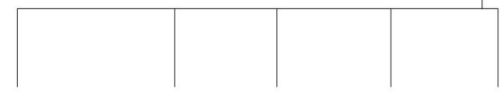

MaxVar Canton-S Oregon-R Berlin-K w1118

\section{WITHIN STRAIN $\longrightarrow$}

\section{Stock Isogenic}

\section{WITHIN \& BETWEEN}
INDIVIDUAL
15 individuals over 3 days

$$
(N=45)
$$

Genetic heterogen

(high to low)
Overall activity proportions

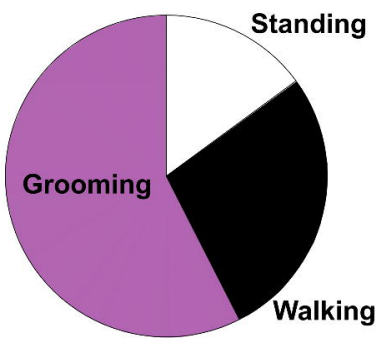

Grooming progression

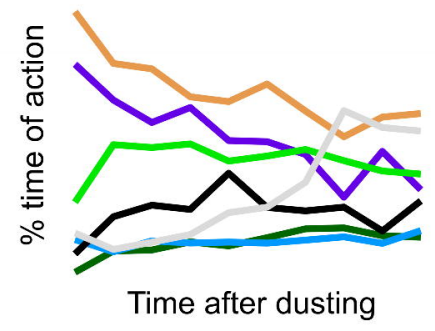




\section{Time spent grooming after dusting}

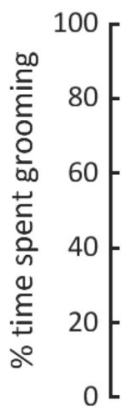
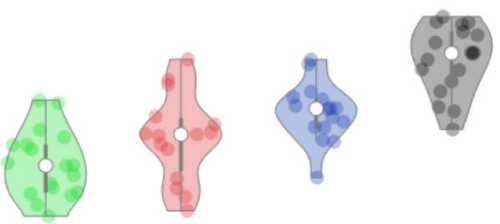

- erecta sechellia osantomea simulans
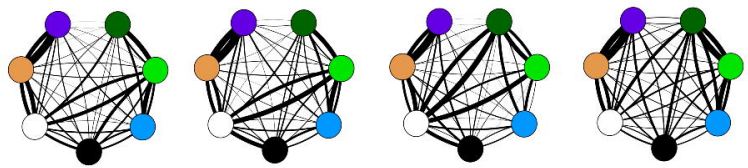

Front le cleaning

\section{Stan}

Low-dimensional embedding (t-SNE) of grooming syntax

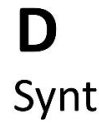

erecta

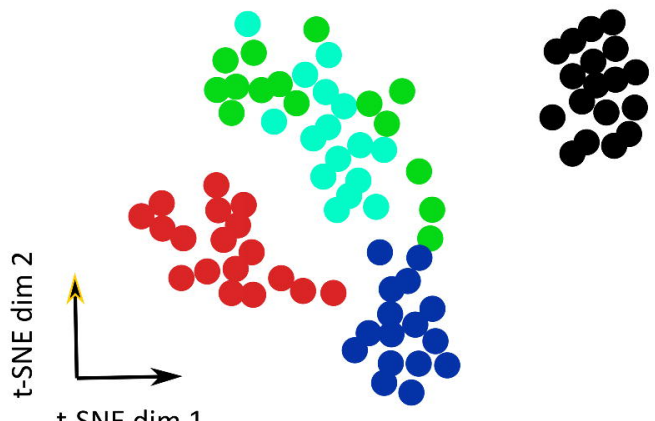

santomea

sechellia

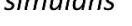


A Melanogaster grooming syntax ( $\mathrm{t}$-SNE embedding)

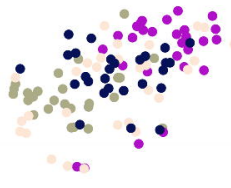

Canton-S Oregon-R

B

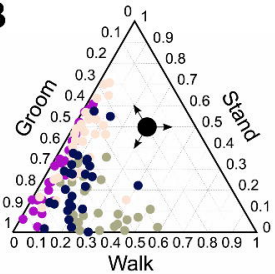

Wing grooming to walking

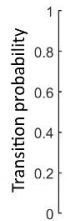

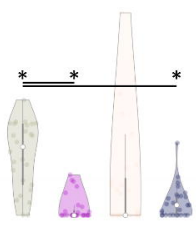

Berlin-K

w1118

Wing grooming to back leg cleaning
Classifier performance (syntax)

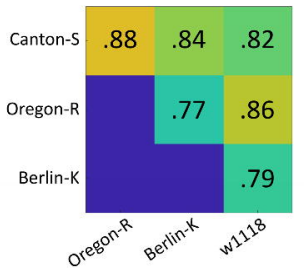

Classifier performance (progressions)
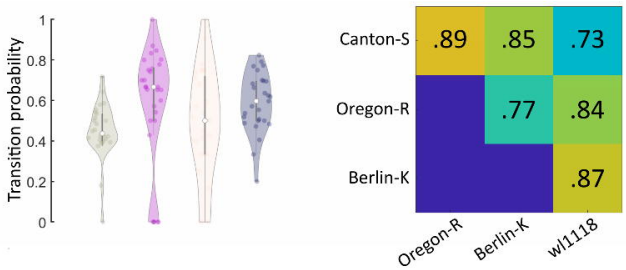

c

름

Abdomen grooming to back leg cleaning (all stocks and isogenized lines)
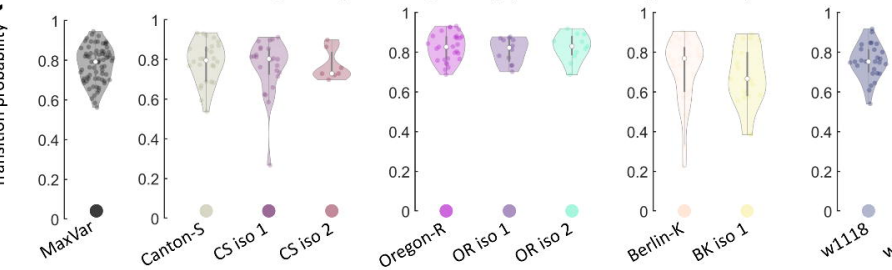

ores or op 
